\title{
Editorial
}

\section{Point Sources and Projection Microscopy}

The $39^{\text {th }}$ Field Emission Symposium took place in Halifax, Nova Scotia, Canada, from August $10^{\text {th }}$ to $14^{\text {th }}, 1992$. It was organized by Pr. J. Kreuzer from Dalhousie University, Halifax. Conferences were held in this University. Most sessions were related to field emission like field electron and field ion microscopies or atom probe studies. Two sessions were about scanning tunneling microscopy, one about diffusion and adsorption processes and one about point sources. It is this particular aspect that I would like to emphasize because of its potential application in the design of new kinds of microscopes.

H.W. Fink, from IBM Zürich detailed applications of electron point sources. He first defined what a point source of particles of wave nature is and how it is possible to build such sources for electrons using ultra-sharp field emission tips. He then showed how the electron beam generated by these sources allows to obtain low-energy electron Gabor type holograms of low $\mathrm{Z}$ atoms or atomically resolved lattice images of thin films. He presented some preliminary results concerning a simple electrostatic microlens set-up used in combination with a point source. In such a configuration a hologram is preserved, albeit distorted, after the focus point of the lens, illustrating the phase difference conservation passing through the lens with a beam opening of several degrees.

R. Morin, from CRMC2 Marseille, presented low energy projection micrographs and holograms and conventional high energy transmission electron micrographs taken on exactly the same objects consisting of thin perforated carbon membranes partly covered by a $5 \mathrm{~nm}$ layer of gold. He reported measurements of a fringe spacing on a wide range of energy (between 16 and $70 \mathrm{eV}$ ) and showed how this supports the holographic interpretation of the observed interference pattern. He also showed how these results allow to measure the increase of electron mean free path with decreasing energy in this low energy range. The sensitivity of low energy electron projection microscopy was illustrated by the obtainment of a hologram of a $3 \mathrm{~nm}$ Au crystal and a hologram down to an ultra-low energy of $7 \mathrm{eV}$ was reported.

The third talk concerning point sources and projection microscopy was given by $\mathrm{H}$. Schmid from IBM Zürich in the frame of the "E.W. Müller Outstanding Young Scientist Competition" and obtained the award. It was about combined electron and ion projection microscopies. A high ionization rate above a single atom tip was achieved with a locally increased gas supply of $\mathrm{He}$ or $\mathrm{H}_{2}$ in a low temperature environment. The effect of the much shorter wavelength of ions compared to that of electrons was beautifully illustrated by looking at the projection pattern of two nearby small holes of a thin perforated membrane, patterns which exhibit fringes only with electrons. Some results concerning ion micromachining and charging-neutralization cycles were also presented.

Roger Morin

CRMC2/CNRS

Campus de Luminy, Marseille 